\title{
Advances in the science of light
}

\author{
Jelena Radovanović ${ }^{1} \cdot$ Mauro F. Pereira ${ }^{2} \cdot$ Dragan Indjin $^{3}$ • \\ Mikhail Sumetsky ${ }^{4} \cdot$ Milutin Stepić $^{5}$
}

Received: 7 July 2016/Accepted: 8 July 2016/Published online: 1 August 2016

(C) Springer Science+Business Media New York 2016

The fifth International School and Conference on Photonics-Photonica 2015 held on 24 August-28 August 2015 in Belgrade, Serbia, was one of the largest events dedicated to the celebration of the UNESCO proclaimed International year of light and light based technologies (IYL 2015) in southern Europe. In a year in which many magnificent scientific anniversaries related to great achievements in light science from the last thousand years coincided, the Vinča Institute of Nuclear Sciences (Belgrade, Serbia), the Serbian Academy of Sciences and Arts, the Optical Society of Serbia and Aston University (Birmingham, UK) tried to contribute to this global initiative which highlighted to the mankind the immense importance of light and related technologies in everyday life by organizing this respectable scientific and educational meeting.

The conference was attended by a record number of 222 registered participants where, for the first time, domestic researchers have been outnumbered by foreign ones from 30 countries and all continents. Almost eighty $\mathrm{PhD}$ and master students had the luck and privilege to get special postal envelopes dedicated to the IYL 2015 and to listen to six illuminating tutorial talks, seven red-hot keynote talks and 21 invited lectures from

This article is part of the Topical Collection on Advances in the Science of Light.

Guest Edited by Jelena Radovanović, Milutin Stepić, Mikhail Sumetsky, Mauro Pereira and Dragan Indjin.

Milutin Stepić

mstepic@vin.bg.ac.rs

1 School of Electrical Engineering, University of Belgrade, Bulevar Kralja Aleksandra 73, Belgrade, Serbia

2 Materials and Engineering Research Institute, Sheffield Hallam University, Sheffield S1 1WB, England, UK

3 School of Electronic and Electrical Engineering, University of Leeds, Leeds LS2 9JT, England, UK

4 Aston Institute of Photonic Technologies, Aston University, Birmingham B4 7ET, England, UK

5 Vinča Institute of Nuclear Sciences, University of Belgrade, P.O.B: 522, Belgrade, Serbia 
outstanding scientists whose trendsetting research already shapes our understanding of optics and photonics.

In a cozy atmosphere of the Serbian Academy of Sciences and Arts, participants could enjoy in two crowded poster sessions and brilliant speeches given by Giulio Cerullo (Politechnico di Milano, Italy), Vlatko Vedral (University of Oxford, UK), Peter Schaaf (Ilmenau University of Technology, Germany), Philip Russell (Max Planck Institute for the Science of Light, Germany), Sandro Stringari (Università di Trento, Italy), Philippe Grangier (CNRS, Universite’ Paris-Sud, France), Alex Rozhin (Aston University, UK), Mario Silveirinha (University of Coimbra, Portugal), Timothy Wilkinson (University of Cambridge, UK), Ortwin Hess (Imperial College London, UK), Edik Rafailov (Aston University, UK), Dragomir Neshev (Australian National University Canberra, Australia), Alexander Szameit (University Jena, Germany) and Stefan Wabnitz (University of Brescia, Italy), to mention only a few.

During the conference, supported by the European Physical Society, the Optical Society of America, European Cooperation in Science and Technology (COST) and the Serbian Ministry of Education, Science and Technological Development, the open-round table "Editors et altera" has been organized. Rachel Won (Nature Photonics), Amos Martinez (Nature Communications), Peter Schaaf (Applied Surface Science) and Mikhail Sumetsky (Optics Letters) presented editorial and publishing policies of their journals and shared their experiences with the audience. Along with the main program, several parallel sessions have been dedicated to the joint COST Actions BM1205, European Network for Skin Cancer Detection using Laser Imaging, MP1204, TERA-MIR Radiation: Materials, Generation, Detection and Applications and the Second international workshop Control of light and matter waves propagation and localization in photonic lattices.

This topical collection of Optical and Quantum Electronics contains a selection of 44 peer reviewed papers that were presented at Photonica 2015. As the conference topics cover a broad range of research activities, we hope that the readers interested in quantum optics, nonlinear optics, ultrafast phenomena, laser spectroscopy, devices and components, biophotonics, optical communication, sensing: plasmonics, fiber sensors, interferometers, holography and adaptive optics and optical materials will find this collection interesting and valuable.

The team of guest editors would like to thank the authors who submitted their papers to the special issue and the reviewers for their often underappreciated work and prompt response. Finally, we would like to thank the members of the conference's Program and Organizing committees on their enthusiasm, members of the OQE editorial team (Trevor Benson, Neeraja Prabakaran, Bhavani Sridhar and Sarvagnan Subramanian) and our sponsors (http://www.vin.bg.ac.rs/photonica2015/sekcije.php?r=sbr-7/OrganisersSponsors.html), without whose inestimable help this topical collection would not be possible.

Guest editors 